Revue de droit comparé du travail et de la sécurité sociale

$3 \mid 2019$

Les migrations internationales de travail

\title{
La réforme des congés payés dans le secteur privé
}

Maria Katia Garcia Landaburu

\section{(2) OpenEdition}

Journals

Édition électronique

URL : https://journals.openedition.org/rdctss/1512

DOI : $10.4000 /$ rdctss. 1512

ISSN : 2262-9815

Éditeur

Centre de droit comparé du travail et de la sécurité sociale

Édition imprimée

Date de publication : 1 novembre 2019

Pagination : 172-175

ISSN : 2117-4350

Référence électronique

Maria Katia Garcia Landaburu, «La réforme des congés payés dans le secteur privé », Revue de droit comparé du travail et de la sécurité sociale [En ligne], 3 | 2019, mis en ligne le 01 novembre 2021, consulté le 13 novembre 2021. URL : http://journals.openedition.org/rdctss/1512 ; DOI : https:// doi.org/10.4000/rdctss. 1512

Revue de droit comparé du travail et de la sécurité sociale est mise à disposition selon les termes de la Licence Creative Commons Attribution - Pas d'Utilisation Commerciale - Pas de Modification 4.0 International. 


\section{LA RÉFORME DES CONGÉS PAYÉS DANS LE SECTEUR PRIVÉ}

Le décret suprême 002-2019-TR, publié le 5 février 2019 dans le journal officiel El Peruano, a approuvé le règlement du décret législatif $n^{\circ} 1405$ dont les dispositions visent, dans le cadre des congés payés, à favoriser la conciliation entre vie professionnelle et familiale des travailleurs rattachés au régime général du secteur privé (les régimes spéciaux du travail étant régis selon leurs propres règles). Si ces nouvelles règles n'apportent aucune modification quant au nombre de jours de congés, maintenu à 30 jours par année travaillée (quels que soient le poste ou l'ancienneté du travailleur), elles ont assoupli la manière dont les travailleurs peuvent aujourd'hui prendre ces congés.

II convient de rappeler que les dispositions relatives aux congés payés ont été établies il y a plus de 25 ans (décret législatif $n^{\circ} 713$ de 1991 et ses règlements, décret suprême 012-92-TR de 1992). Bien qu'à l'époque ces règles aient permis de simplifier le régime des congés, en regroupant des dispositions isolées qui réglementaient à la fois les employés, les ouvriers, et qui dataient de plusieurs décennies (plus précisément de 1933), elles se sont avérées obsolètes et inadaptées aux besoins de l'ensemble des travailleurs.

Pour apprécier ces changements, la présente contribution examinera le régime des congés initialement établi par le décret législatif $n^{\circ} 713$ et ses règlements (I), avant d'analyser les évolutions mises en œuvre il y a quelques mois aux niveaux légal et réglementaire (II), à l'origine de la révision des articles 10,17 et 19 du décret législatif $n^{\circ} 713$ (ci-après « la Loi ») et d'une règle complémentaire au décret suprême 012-92-TR.

\section{I - LE RÉGIME DES CONGÉS DANS LE DÉCRET Nº 713}

La Loi et ses règlements ont notamment établi ce qui suit:

- Les travailleurs ont droit à 30 jours calendaires de congés par année complète travaillée, à condition de remplir le quota de jours travaillés légalement requis, ce qui équivaut pour des travailleurs ayant un rythme de travail normal de 5 jours par semaine à 210 jours de travail effectifs dans l'année (article 10 de la Loi).

- L'employeur et le travailleur fixent d'un commun accord le moment opportun pour prendre des congés, en tenant compte des besoins opérationnels de l'entreprise et de l'intérêt du travailleur. En l'absence d'accord, la décision revient à l'employeur, dans le cadre de l'exercice de ses fonctions de direction (article 14 de la Loi).

- Le travailleur doit bénéficier de ses congés de manière continue. Toutefois, sur demande écrite du travailleur, l'employeur peut l'autoriser à prendre des congés de manière fractionnée dès lors que la durée de chaque période n'est pas inférieure à 7 jours calendaires (article 17 de la Loi).

- Le travailleur peut convenir par écrit avec son employeur de cumuler jusqu'à deux périodes de congés consécutifs, à condition qu'après une année travaillée continue, il bénéficie d'au moins une période de congés de 7 jours calendaires. Dans le cas des travailleurs engagés 
à l'étranger, ils peuvent convenir par écrit de cumuler les périodes de congés de deux ans ou plus (article 18 de la Loi).

- Les congés peuvent être réduits de 30 à 15 jours, moyennant une indemnité équivalente à 15 jours de rémunération (le droit de bénéficier des 15 jours de congés restants est inaliénable). L'accord de réduction doit être écrit (article 19 de la Loi).

- Si le travailleur ne bénéficie pas de ses congés dans l'année suivant celle où il en a acquis le droit, il peut prétendre: soit à la rémunération pour le travail effectué (normalement versée pour le mois travaillé lorsque les congés n'ont pas été pris), soit à une rémunération pour les congés annuels acquis et non pris, soit à une indemnité équivalente à la rémunération pour ne pas avoir bénéficié des congés.

- Le montant des rémunérations indiquées correspond à celui que le travailleur reçoit au moment du paiement (article 23 de la Loi).

- L'indemnité prévue en cas de congés non pris ne s'applique pas aux dirigeants ou représentants d'entreprise qui ont décidé de ne pas prendre leurs congés annuels (article 24 du décret suprême 012-92-TR).

Si ces règles sont restées inchangées jusqu'à l'année dernière, des discussions au niveau judiciaire ont toutefois porté sur l'indemnité accordée lors de congés non pris, tant en ce qui concerne l'émergence de ce droit que de ses bénéficiaires.

La naissance de ce droit est le résultat d'un fait objectif. Mais la Cour suprême a établi en 1998 un critère assez discutable lors de la résolution du recours en cassation $n^{\circ} 1633-98$, selon lequel l'indemnité prévue par la loi devait être versée dès lors que les congés n'avaient pas été pris, faisant ainsi disparaître ce droit lorsque les congés avaient effectivement été pris, mais avec du retard. Quelques années plus tard, la Cour suprême a modifié ce critère (Cassation n²170-2003 - Lima - du 15 avril 2005), estimant que l'employeur ne se dégage pas du paiement de l'indemnité quand il accorde un repos physique à son travailleur en dehors du délai prévu par la loi, dans la mesure où l'essence même du versement de cette indemnité consiste à permettre au travailleur de se ressourcer lorsqu'il n'a pas bénéficié de congés après une année continue de travail.

\section{II - LES MODIFICATIONS APPORTÉES PAR LE DÉCRET LÉGISLATIF Nº 1405}

Le décret législatif n 1405 s'appuie sur la Loi n³0823, aux termes de laquelle le Congrès de la République délègue à l'Exécutif le pouvoir de légiférer dans les domaines de l'économie, de la compétitivité, de l'intégrité, de la lutte contre la corruption, de la prévention et la protection des personnes exposées à la violence et à la vulnérabilité, de la modernisation de l'État.

Outre la Constitution de 1993, ce décret a pour base juridique deux Conventions de I'Organisation Internationale du Travail ratifiées par le Pérou: i) la Convention n 52, dont l'article 2 permet au législateur national d'autoriser - à titre exceptionnel - le découpage de la période des congés annuels d'une période minimale de 6 jours ouvrables; et ii) la Convention $n^{\circ} 156$ sur les travailleurs ayant des responsabilités familiales, dont l'article 3 engage les États à prendre des objectifs politiques nationaux visant à permettre aux personnes ayant des responsabilités familiales et qui exercent - ou souhaitent exercer - un emploi de le faire sans discrimination, et si possible sans conflit entre leurs responsabilités familiales et professionnelles. 
Ce décret législatif réglemente les congés payés des fonctionnaires pour qu'ils favorisent la conciliation de leur vie professionnelle et familiale, "contribuant ainsi à la modernisation du système administratif de gestion des ressources humaines de l'État ». Des dispositions complémentaires modifient trois articles du décret législatif $n^{\circ} 713$, « conformément au principe constitutionnel de l'égalité devant la loi ». On constate donc que la réforme du secteur privé n'a pas été directe, en raison de la délégation limitée des pouvoirs législatifs.

Dans le paragraphe suivant, seront examinées les modifications apportées à la loi par le décret-loi $n^{\circ} 1405$ et son règlement, approuvées par le décret suprême n002-2019-TR (ci-après « les dispositions réglementaires »).

\section{A - CONGÉS ANTICIPÉS}

L'article 10 de la Loi, tel que modifié, permet désormais, par accord écrit entre les parties, de prendre des congés anticipés qui seront décomptés des jours acquis par la suite. L'article 5 des « dispositions réglementaires » précise que l'accord doit être conclu avant le début des congés et que ces derniers peuvent couvrir un nombre de jours supérieur à celui cumulé à la date de l'accord. Autrement dit, si 2,5 jours de congés sont cumulés par mois travaillé, rien n'empêche, après 6 mois d'activité, de prendre des congés anticipés de 20 ou même 30 jours.

Cette situation est provisoire, dans la mesure où le travailleur aura accumulé des jours de congés après avoir rempli le quota correspondant, lui permettant ainsi de compenser les jours de congés pris de manière anticipée. Si la relation de travail prend fin avant que le quota de jours de congés ne soit atteint, la loi prévoit que les jours de congés accordés de manière anticipée au travailleur peuvent être compensés par les jours de congés restant acquis à la date de cessation, l'indemnité devant être expressément détaillée dans le règlement des prestations sociales. Si des jours de congés anticipés ne peuvent être compensés par les jours de congés restant acquis, le travailleur est déchargé de toute obligation d'indemnité et de paiement (la valeur de ces jours ne peut être déduite des prestations sociales qui lui sont versées).

\section{B - CONGÉS FRACTIONNÉS}

Comme indiqué précédemment, les congés annuels sont fixés à 30 jours calendaires et doivent en principe être pris de manière ininterrompue. Sur demande écrite du travailleur, les congés peuvent être pris comme suit: i) 15 jours calendaires ou plus de manière ininterrompue ou par tranches d'au moins 7 ou 8 jours consécutifs; et ii) les jours de congés restants, qui peuvent être pris à la journée. Les parties peuvent convenir en amont, par écrit, de l'ordre du fractionnement des congés en indiquant expressément les différentes périodes, avec les dates de début et de fin.

La deuxième option, qui permet de prendre les congés à la journée, ne fait pas passer automatiquement le reste des congés en jours ouvrables. Comme le précise l'article 4 des dispositions règlementaires, les congés annuels sont fixés à 30 jours calendaires et " comprennent les jours de repos hebdomadaires, les jours fériés, les jours non ouvrables et autres interruptions du travail pendant la période de congés correspondante, sauf décision unilatérale de l'employeur, accord entre les parties, convention collective ou usage plus favorable». 
L'employeur se réserve bien évidemment le droit d'accepter ou non la demande de fractionnement des congés du travailleur. L'article 7, paragraphe 2, des dispositions réglementaires prévoit que les congés et leur fractionnement sont déterminés d'un commun accord entre l'employeur et le travailleur. A défaut d'accord, l'employeur décide du moment opportun de mise en œuvre des congés, mais pas de leur fractionnement.

\section{C - RÉduCTION DE LA DURÉE DES CONGÉS}

Le nouvel article 19 de la loi maintient la possibilité pour les parties de convenir de la réduction des congés de 30 à 15 jours calendaires, mais adapte son contenu au fait qu'il est désormais possible de prendre les congés à la journée. Il précise donc que la réduction ne peut s'appliquer qu'aux congés qui peuvent être pris en périodes notamment inférieures à 7 jours, de sorte que les congés doivent être pris en continu ou en périodes de 7 ou 8 jours consécutifs. 